MODELING, IDENTIFICATION AND CONTROL, 2000, VOL. 21, NO. 4, 193-208

doi:10.4173/mic.2000.4.1

\title{
A Nonlinear Observer for Integration of GPS and Inertial Navigation Systems
}

\author{
BJØRNAR VIK and THOR I. FOSSEN† \\ Keywords: Navigation, GPSIINS integration, estimation, Lyapunov design, \\ nonlinear analysis
}

\begin{abstract}
GPS and INS have complementary properties and they are therefore well suited for integration. The integrated solution offers better long term accuracy than a stand-alone INS, and better integrity, availability and continuity than a standalone GPS receiver, making it suitable for demanding applications. The integrated filter is nonlinear both in state and measurements, and the extended Kalman-filter has been used with good results. But the extended Kalman-filter has not been proven globally stable, and it is also computationally intensive, especially within a direct integration architecture. In this work exponential stability of the origin of the combined attitude and velocity error systems is proven along with robust stability in the presence of noise and unmodelled dynamics.
\end{abstract}

\section{Introduction}

The complementary features of Inertial Navigation Systems (INS) and the Global Positioning System (GPS) are the main reasons why integrated GPS/INS systems are becoming increasingly popular. The high long term accuracy of GPS can be combined with the high output rate, dynamic capability, robustness, and reliability of INS. In the case of GPS drop-outs, the INS will be calibrated when coasting through the outage. After the outage, the INS can provide estimates of position and velocity to the GPS receiver to speed up reaquisition. GPS/INS systems thercfore offer position, velocity, acceleration, attitude and angular velocity measurements with high accuracy, output rate, and reliability in one package.

Several levels of integration have been mentioned in the literature (see e.g. Phillips and Schmidt (1996), and Greenspan et al. (1988)), among them uncoupled, loose, tight, and deep integration. With increasingly tight coupling, performance and robustness against interference will increase at the cost of increased complexity, possible reduction of redundancy, and somewhat reduced flexibility. An example of a loosely coupled architecture is shown in Figure 1, and an example of a tightly coupled architecture is shown in Figure 2.

The main difference between the architectures is that in tightly coupled systems raw accelerometer, gyro, pseudorange, and deltarange measurements from the sensors are used instead of position, velocity and attitude data. One main advantage of tight integration is that even in the case where less than the necessary amount of satellites are available for a navigation solution, the remaining pseudoranges and deltaranges provide information that will help the INS coast through the outage. Another key feature, at least for military applications, is velocity aiding of the GPS receiver correlator loops. The aiding enables the receiver to keep the bandwidths of the tracking loops low even under highly dynamic conditions, thereby increasing jamming resistance. Tightly coupled systems have also been reported to be more accurate than the

$\dagger$ Norwegian University of Science and Technology. 


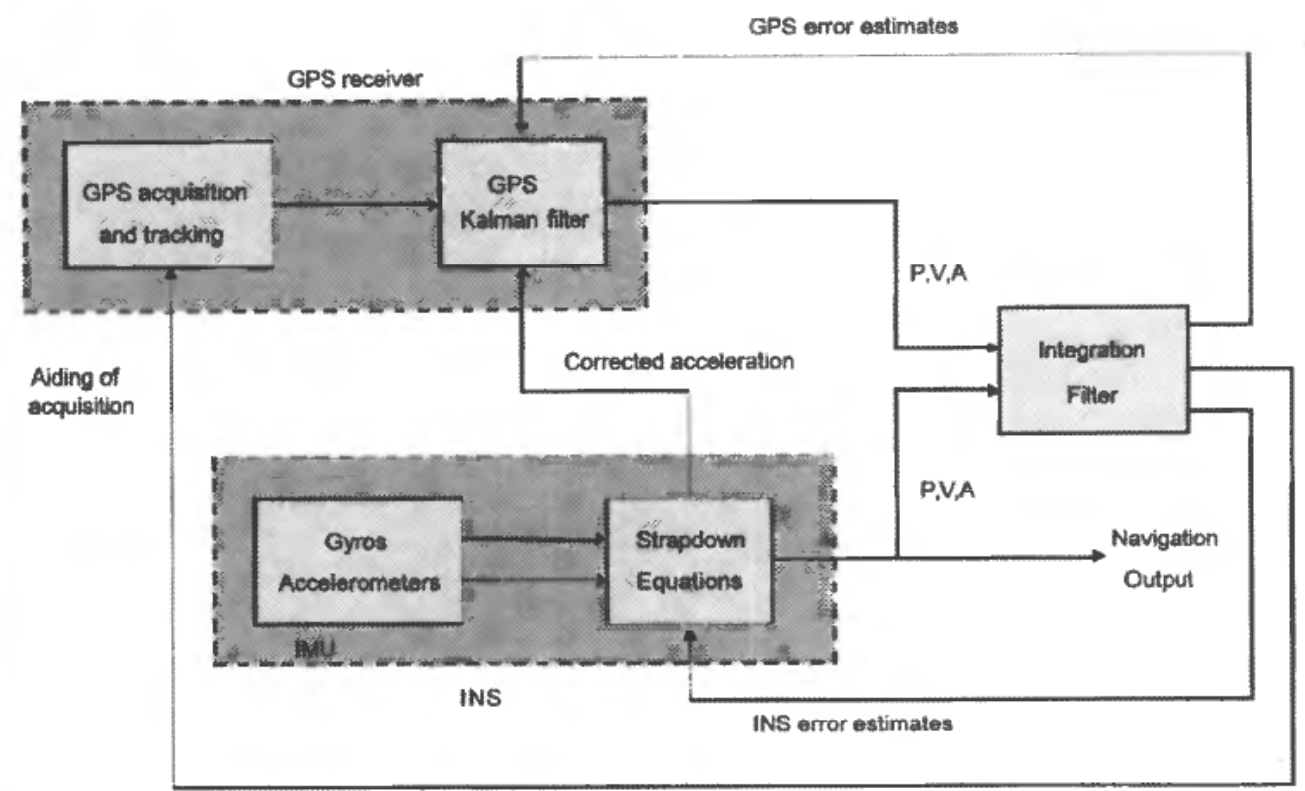

Figure 1. Loosely coupled GPS/INS system.

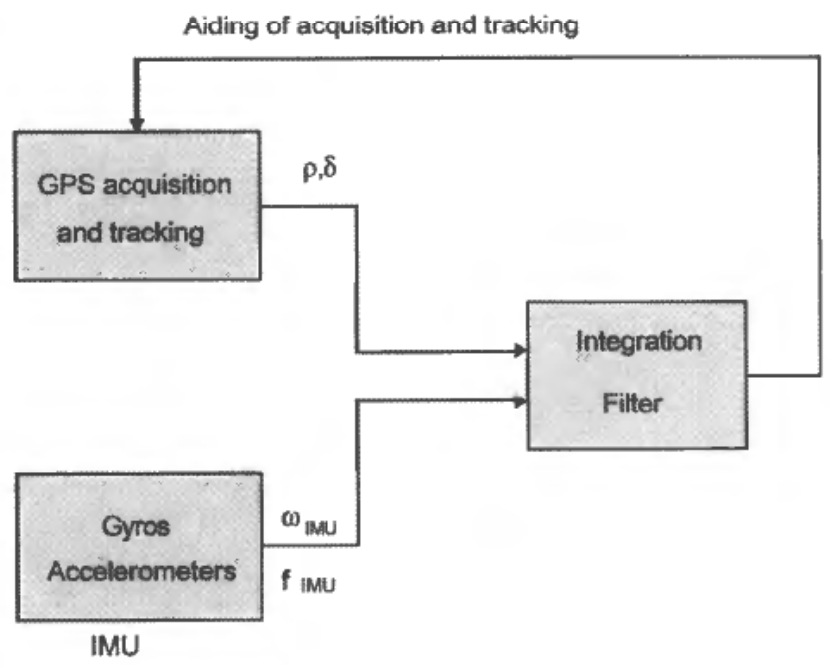

Figure 2. Tightly coupled GPS/INS system.

loosely coupled systems. These advantages comes at the cost of increased complexity and increased demand for computer throughput in the navigation computer due to the fact that both GPS and strapdown software must be implemented in the navigation computer. However, the last few years there has been a trend towards more tightly integrated systems.

In addition to the different levels of integration, there are two different ways of modelling position.velocity and attitude variables, known as direct and indirect integration (Maybeck, 1979). The most popular approach is indirect integration where errors are estimated in the integration filter and used to update the whole state 
variables, like position and velocity, that are output from the strapdown computations. The filter can be designed with a slow update rate due to the slow dynamics of the error model. This approach naturally belongs together with an uncoupled or loosely coupled architecture. The direct approach involves estimating the whole states in the filter. It has been argued that this requires more computer throughput since the dynamics of the whole states is much larger than the dynamics of the error states and subsequently require higher iteration rate. However, in a tightly coupled architecture, the strapdown equations must be performed in the same computer anyway. This argument is therefore only valid if a Kalman filter, where the state covariance matrix must be updated at a high iteration rate, is used.

In this paper, a nonlinear observer that does not require high iteration computations, is presented. Direct integration using this observer is not second to indirect integration when it comes to computer throughput requirements. In fact, since the observer does not include position, velocity and attitude error models, there are fewer computations in comparison to an indirect architecture. The observer, being a direct filter, is intended for systems where IMU data (acceleration and angular velocity) are used directly in the filter. For other systems, the standard error model approach will be more suitable. In Vik et al. (1999), the observer was analyzed using a cascaded approach, and quasi-equi asymptotic stability (QEAS) of the error system origin was proven (see Lakshmikantham et al. (1991) for a definition of QEAS). In this paper, we prove global exponential stability (GES) of the origin. This is a stronger result than QEAS.

\section{Notation}

When dealing with several coordinate frames, it is necessary to know which frame the vectors are decomposed into. We will refer to the different coordinate systems using the indexes of Table 1.

Vectors are written with a superscript showing which frame the vector is decomposed into. For example:

$$
v^{n}=\left[v_{N}, v_{E}, v_{D}\right]^{T}: \text { Platform velocity decomposed into the NED frame }
$$

The relative angular velocity between two frames is described using angular velocity vectors. These vectors will, in addition to superscripts, have subscripts showing which two frames that are rotating relative to each other. For example:

$\omega_{i e}^{n} \in \mathbb{R}^{3}$ : Angular velocity of ECI relative to ECEF decomposed into the NED frame

Addition of two angular velocity vectors are reflected in the notation by cancelling the inner subscript as follows:

$$
\omega_{i n}^{n}=\omega_{i e}^{n}+\omega_{e n}^{n}
$$

Table 1. Coordinate system indexes

\begin{tabular}{llc}
\hline Index & \multicolumn{1}{c}{ Coordinate system } & Components \\
\hline$i$ & Earth Centered Inertial (ECI) & $x_{i}, y_{i}, z_{i}$ \\
$e$ & Earth Centered Earth Fixed (ECEF) & $x_{e}, y_{e}, z_{e}$ \\
$n$ & North, East, Down (NED) & $N, E, D$ \\
$b$ & BODY & $x_{b}, y_{b}, z_{b}$ \\
\hline
\end{tabular}


Table 2. Overview over vectors used in this paper

\begin{tabular}{lll}
\hline Frame & \multicolumn{1}{c}{ Vectors } & \multicolumn{1}{c}{ Comments } \\
\hline ECEF & $\mathbf{r}^{e}=\left[x_{e}, y_{e}, z_{e}\right] \in \mathbb{R}^{3}$ & Cartesian ECEF position \\
NED & $\mathbf{v}^{n}=\left[v_{N}, v_{E}, v_{D}\right] \in \mathbb{R}^{3}$ & North, east and down velocity \\
NED/ECEF & $\omega_{e n}^{n}=\left[\omega_{N}, \omega_{E}, \omega_{D}\right] \in \mathbb{R}^{3}$ & Function of vehicle velocity \\
BODY/NED & $\omega_{n b}^{b}=[p, q, r] \in \mathbb{R}^{3}$ & Roll, pitch and yaw rate \\
ECEF/ECI & $\omega_{i e}^{e}=\left[0,0, \omega_{\epsilon}\right] \in \mathbb{R}^{3}$ & $\omega_{\epsilon}$ is angular velocity of Earth \\
\hline
\end{tabular}

The order of the subscript is reversed when the direction of the rotation is changed, i.e.

$$
\omega_{i n}^{n}=-\omega_{n i}^{n}
$$

Rotation of a vector is written:

$$
\mathbf{v}^{n}=\mathbf{R}_{b}^{n} \mathbf{v}^{b}
$$

where the BODY-fixed velocity $\mathbf{v}^{b}$ is decomposed into the NED frame by multiplication with the rotation matrix $\mathbf{R}_{b}^{n}$.

Cross products will be written in matrix form using the skew-symmetrical matrix operator:

$$
S(\omega)=[\omega \times]
$$

The most common translatory and rotational vectors that will be used below is summarized in Table 2.

\section{Strapdown Equations}

Position, velocity and attitude variables from an INS are derived from measurements of acceleration nominally made along the three orthogonal axes in the BODY frame, and from angular velocity (gyroscope) measurements of rotation of the inertial frame relative to the BODY frame. For applications on the Earth, the inertial frame can be well approximated by the ECI frame. The GPS measurements are given in the ECEF frame, while the IMU measurements are given in the body frame. The best choice of reference frame for integration is dependent from the application and the priorities of the system integrator. In this paper the observer velocities will be given in the NED frame, while positions are given in the ECEF frame. Longitude and latitude can easily be calculated from ECEF positions using well known algorithms, see e.g. Hofmann-Wellenhof $e t$ al. (1994).

\section{Attitude equations}

The attitude will be represented using the singularity free unit quaternion $\mathbf{q} \in \mathbb{H}$, where

$$
\mathbb{H}=\left\{\mathbf{q} \mathbf{q}^{T} \mathbf{q}=1, \mathbf{q}=\left[\boldsymbol{\eta}, \varepsilon^{T}\right]^{T}, \boldsymbol{\eta} \in \mathbb{R}, \varepsilon \in \mathbb{R}^{3}\right\}
$$

The differential equations describing rotation of the BODY frame relative to the NED frame is:

$$
\dot{\mathbf{q}}=\left[\begin{array}{c}
\dot{\eta} \\
\dot{\varepsilon}
\end{array}\right]=\frac{1}{2}\left[\begin{array}{c}
-\varepsilon^{T} \\
\eta \mathbf{I}+\mathbf{S}(\varepsilon)
\end{array}\right] \omega_{n b}^{b}
$$


Error free gyros measure the rotation of the BODY frame relative to the ECI frame decomposed into the BODY frame, i.e. $\omega_{i b}^{b}$. Thus, $\omega_{n b}^{b}$ can be found from:

$$
\omega_{n b}^{b}=\omega_{i b}^{b}-\omega_{i n}^{b}=\omega_{i b}^{b}-\left(\omega_{i e}^{b}+\omega_{e n}^{b}\right)
$$

Applying (2) and (3) gives the unit quaterion differential equations:

$$
\begin{aligned}
{\left[\begin{array}{l}
\dot{\eta} \\
\dot{\varepsilon}
\end{array}\right] } & =\frac{1}{2}\left[\begin{array}{c}
-\varepsilon^{T} \\
\eta \mathbf{I}+\mathbf{S}(\varepsilon)
\end{array}\right] \omega_{i b}^{b}-\frac{1}{2}\left[\begin{array}{c}
-\varepsilon^{T} \\
\eta \mathbf{I}-\mathbf{S}(\varepsilon)
\end{array}\right]\left(\omega_{i e}^{n}+\omega_{e n}^{n}\right) \\
& =\frac{1}{2} \mathbf{T}_{\mathbf{q}}(\mathbf{q}) \omega_{i b}^{b}-\frac{1}{2} \Xi(\mathbf{q})\left(\omega_{i e}^{n}+\omega_{e n}^{n}\right)
\end{aligned}
$$

where matrices $\mathbf{T}_{\mathbf{q}}(\mathbf{q})$ and $\Xi(\mathbf{q})$ has been introduced for easy reading. $\omega_{i e}^{n}$ and $\omega_{e n}^{n}$ can be calculated from (12) and (11), respectively.

\section{Position and velocity equations}

The NED frame is the most popular mechanization for navigation systems for applications on and close to the surface of Earth. The differential equations for NED frame velocity can be found in e.g. Britting (1971):

where

$$
\dot{\mathbf{v}}^{n}=\mathbf{R}_{b}^{n} \mathbf{f}^{b}+\mathbf{g}^{n}-\left[2 \mathbf{S}\left(\omega_{i e}^{n}\right)+\mathbf{S}\left(\omega_{e n}^{n}\right)\right] \mathbf{v}^{n}
$$

$$
\mathbf{f}^{b}=\mathbf{R}_{n}^{b}\left(\overline{\mathbf{g}}^{n}-\mathbf{S}^{2}\left(\omega_{i e}^{n}\right) \mathbf{r}^{n}\right)
$$

is the specific force measurements made by the accelerometers. $\overline{\mathbf{g}}^{n}$ is gravity due to the mass of the Earth, while

$$
\mathbf{g}^{n} \triangleq \overline{\mathbf{g}}^{n}-\mathbf{S}^{2}\left(\omega_{i e}^{n}\right) \mathbf{r}^{n}
$$

is referred to as plumb bob gravity since a plumb bob at rest would be directed along the accelerometer sensed line of force. The sum of the mass attrction gravity term plus the centripetal term resulting from Earth rotation will therefore be directed along the down axis, i.e.

$$
\mathbf{g}^{n}=\left[\begin{array}{lll}
0 & 0 & g_{D}
\end{array}\right]^{T}
$$

where $g_{D}$ can be assumed known with sufficient accuracy (below the noise floor of the accelerometers) for most systems. In some high accuracy applications, errors in gravity magnitude as well as direction are estimated. For calculation of position, several representations can be used. Often, the system is divided into horizontal (latitude and longitude) and vertical (height) channels. The differential equations are:

$$
\begin{array}{r}
\dot{h}=\mathbf{c}^{T} \mathbf{v}^{n}=-v_{D} \\
\dot{\mathbf{R}}_{e}^{n}=-\mathbf{S}\left(\omega_{e n}^{n}\right) \mathbf{R}_{e}^{n}
\end{array}
$$

where

$$
\mathbf{c}=\left[\begin{array}{lll}
0 & 0 & -1
\end{array}\right]^{T}
$$

Latitude and longitude can be calculated from the rotation matrix $\mathbf{R}_{n}^{e}$. Position can also be expressed in ECEF Cartesian coordinates:

$$
\dot{\mathbf{r}}^{e}=\mathbf{R}_{n}^{e} \mathbf{v}^{n}
$$


Formula (9) is useful for integration with GPS, where position measurements are given as Cartesian ECEF coordinates. Latitude, longitude, and height can then be calculated from $\mathbf{r}^{e}$. In this paper, the latter representation will be used. The total position/velocity system can now be written:

$$
\begin{aligned}
\dot{\mathbf{v}}^{n} & =\mathbf{R}_{b}^{n} \mathbf{f}^{b}+\mathbf{g}^{n}-\left[2 \mathbf{S}\left(\omega_{i e}^{n}\right)+\mathbf{S}\left(\omega_{e n}^{n}\right)\right] \mathbf{v}^{n} \\
\dot{\mathbf{r}}^{e} & =\mathbf{R}_{n}^{e} \mathbf{v}^{n}
\end{aligned}
$$

For implementation of (10a) and (10b), $\omega_{e n}^{n}$ can be calculated directly from the NED velocities using the following equations:

$$
\omega_{e n}^{n}=\left[\begin{array}{c}
\omega_{N} \\
\omega_{E} \\
\omega_{D}
\end{array}\right]=\left[\begin{array}{c}
\frac{1}{r_{l}} v_{E} \\
-\frac{1}{r_{\mu}} v_{N} \\
-\frac{1}{r_{l}} v_{E} \tan \mu
\end{array}\right] \triangleq \mathbf{U} \mathbf{v}^{n}
$$

where

$$
\mathbf{U}=\left[\begin{array}{ccc}
0 & \frac{1}{r_{l}} & 0 \\
-\frac{1}{r_{\mu}} & 0 & 0 \\
0 & \frac{1}{r_{l}} \tan \mu & 0
\end{array}\right]
$$

$r_{\mu}$ and $r_{l}$ are the north and east radii of curvature of the local horizontal.

The angular velocity $\omega_{i e}^{n}$ can be calculated from:

$$
\omega_{i e}^{n}=\mathbf{R}_{e}^{n} \omega_{i e}^{e}
$$

where $\omega_{i e}^{e}=\left[0,0, \omega_{e}\right]^{T}$ is known.

Note that by inserting (11) into (10a), a quadratic nonlinearity in $\mathbf{v}^{n}$ is introduced. Also, $\mathbf{R}_{n}^{e}$ is a nonlinear function of $\mathbf{r}^{e}$. The effects of these nonlinearities are small compared to the nonlinearities associated with $\mathbf{R}_{b}^{n}$. In fact, the skew-symmetrical terms in (10a) are only important during long GPS outages, in which case the nonlinear stability analyses presented below are invalid. The skew symmetrical terms and $\mathbf{R}_{n}^{e}$ can thus be assumed constant and known in the stability analyses.

It should be mentioned that for applications with longer than a few minutes operational time, some kind of external height measurement is required to stabilize the vertical channel. For marine applications a height constraint can be applied, e.g. zero sea level. Otherwise, a barometric altimeter is typically applied. GPS measurements can also be applied, but it will still be of interest to have some other measurement available in the case of long duration GPS dropouts.

\section{IMU error models}

The most dominating errors are usually biases, scale-factor errors and misalignment errors. The biases and scale-factor errors are usually precompensated through 
calibration and thermometer fcedback, and the misalignment errors (which include non-orthogonality of the sensor axes) are made as small as possible during mounting of the IMU. However, there are still residual errors that need to be estimated. The output of the IMU can be modelled as:

$$
\begin{aligned}
\omega_{\mathrm{imu}} & =[\mathbf{I}-\Delta(\kappa, \alpha)] \omega_{i b}^{b}-\mathbf{b}_{1}-\mathbf{w}_{1} \\
\mathbf{f}_{\mathrm{imu}} & =[\mathbf{I}-\Delta(\varepsilon, \boldsymbol{\beta})] \mathbf{f}^{b}-\mathbf{b}_{\mathbf{2}}-\mathbf{w}_{\mathbf{5}}
\end{aligned}
$$

where

$$
\Delta=\Delta(\mathbf{s}, \phi)=\left[\begin{array}{ccc}
s_{x} & \phi_{x y} & \phi_{x z} \\
\phi_{y x} & s_{y} & \phi_{y z} \\
\phi_{z x} & \phi_{z y} & s_{z}
\end{array}\right]
$$

Here $\mathbf{s}=\left[s_{x}, s_{y}, s_{z}\right]^{T}$ are three scale factor errors and

$$
\phi=\left[\phi_{x y}, \phi_{x z}, \phi_{y x}, \phi_{y z}, \phi_{z x}, \phi_{z y}\right]^{T}
$$

are six small misalignment angles. $\mathbf{b}_{1} \in \mathbb{R}^{3}$ and $\mathbf{b}_{2} \in \mathbb{R}^{3}$ represent gyro and accelerometer biases, respectively. $\mathbf{w}_{1} \in \mathbb{R}^{3}$ and $\mathbf{w}_{5} \in \mathbb{R}^{3}$ are bounded unmodeled errors and measurement noise. Since the errors are small, the following approximation can be made:

$$
\begin{aligned}
\omega_{i b}^{b} & =[\mathbf{I}-\Delta(\kappa, \alpha)]^{-1}\left(\omega_{\mathrm{imu}}+b_{1}+w_{1}\right) \\
& \approx[\mathbf{I}+\Delta(\kappa, \alpha)]\left(\omega_{\mathrm{imu}}+b_{1}+w_{1}\right) \\
& \approx[\mathbf{I}+\Delta(\kappa, \alpha)] \omega_{\mathrm{imu}}+b_{1}+w_{1}
\end{aligned}
$$

where higher order terms are removed. This approximation is made in order to make the nonlinear analysis easier. Thus, the IMU errors will be modelled as:

$$
\begin{aligned}
\omega_{i b}^{b} & =[\mathbf{I}+\Delta(\kappa, \alpha)] \omega_{\mathrm{imu}}+\mathbf{b}_{1}+\mathbf{w}_{1} \\
\mathbf{f}^{b} & =[\mathbf{I}+\Delta(\varepsilon, \beta)] \mathbf{f}_{\mathrm{imu}}+\mathbf{b}_{\mathbf{2}}+\mathbf{w}_{5}
\end{aligned}
$$

Notice that all error signals are decomposed in the body frame. The magnitude of these errors are in general directly proportional to the price of the IMU. The gyro error models are assumed to be described by the 1st-order models:

$$
\begin{gathered}
\dot{b}_{1}=-T_{1}^{-1} b_{1}+w_{2} \\
\dot{\boldsymbol{k}}=-T_{2}^{-1} \boldsymbol{\kappa}+\mathbf{w}_{3} \\
\dot{\alpha}=-\mathbf{T}_{3}^{-1} \alpha+\mathbf{w}_{4}
\end{gathered}
$$

driven by Gaussian white noise signals $\mathbf{w}_{2}, \mathbf{w}_{3} \in \mathbb{R}^{3}$ and $\mathbf{w}_{4} \in \mathbb{R}^{6}$. The matrices $\mathbf{T}_{1}, \mathbf{T}_{2} \in \mathbb{R}^{3 \times 3}$ and $\mathbf{T}_{3} \in \mathbb{R}^{6 \times 6}$ are diagonal matrices of time constants, $\boldsymbol{\kappa}=\left[\kappa_{x}, \kappa_{y}, \kappa_{z}\right]^{T}$ are three gyro scale factor errors, and $\alpha=\left[\alpha_{x y}, \alpha_{x z}, \alpha_{y x}, \alpha_{y z}, \alpha_{z x}, \alpha_{z y}\right]^{T}$ are six small gyro misalignment angles. The accelerometer errors are described accordingly:

$$
\begin{aligned}
\dot{b}_{2} & =-T_{4}^{-1} b_{2}+w_{6} \\
\dot{\varepsilon} & =-T_{5}^{-1} \varepsilon+w_{7} \\
\dot{\beta} & =-T_{6}^{-1} \beta+w_{8}
\end{aligned}
$$




\section{GPS/INS Attitude and Velocity Observer}

By combining (5), (15) and (17), the error free system can be written:

$$
\begin{aligned}
& {\left[\begin{array}{l}
\dot{\eta} \\
\dot{\varepsilon}
\end{array}\right]=\frac{1}{2} \mathbf{T}_{\mathbf{q}}(\mathbf{q})\left[(\mathbf{I}+\Delta(\kappa, \alpha)) \omega_{\mathrm{imu}}+\mathbf{b}_{1}+\mathbf{w}_{1}\right]-\frac{1}{2} \Xi(\mathbf{q})\left(\omega_{i e}^{n}+\omega_{e n}^{n}\right)} \\
& \dot{b}_{1}=-T_{1}^{-1} \mathbf{b}_{1}+\mathbf{w}_{2} \\
& \dot{\boldsymbol{\kappa}}=-\mathbf{T}_{2}^{-1} \boldsymbol{\kappa}+\mathbf{w}_{3} \\
& \dot{\omega}=-\mathbf{T}_{3}^{-1} \alpha+\mathbf{w}_{4}
\end{aligned}
$$

The following attitude observer is proposed (Vik 2000):

$$
\begin{aligned}
& \dot{\hat{\mathbf{q}}}=\frac{1}{2} \mathbf{T}_{\mathbf{q}}(\hat{\mathbf{q}}) \tilde{\mathbf{R}}\left[\left(\mathbf{I}+\hat{\mathbf{\Delta}}_{1}\right) \omega_{\mathrm{imu}}+\hat{\mathbf{b}}_{1}+\mathbf{K}_{1} \tilde{\varepsilon} \operatorname{sgn}(\tilde{\eta})\right]-\frac{1}{2} \Xi(\hat{\mathbf{q}}) \omega_{i n}^{n} \\
& \dot{\hat{\mathbf{b}}}_{1}=-\mathbf{T}_{1}^{-1} \hat{\mathbf{b}}_{1}+\frac{1}{2} \mathbf{K}_{2} \tilde{\varepsilon} \operatorname{sgn}(\tilde{\eta}) \\
& \dot{\hat{\boldsymbol{\kappa}}}=-\mathbf{T}_{2}^{-1} \hat{\boldsymbol{\kappa}}+\frac{1}{2} \mathbf{K}_{3} \operatorname{diag}(\tilde{\varepsilon}) \omega_{\mathrm{imu}} \operatorname{sgn}(\tilde{\eta}) \\
& \dot{\hat{\alpha}}=-\mathbf{T}_{3}^{-1} \hat{\alpha}+\frac{1}{2} \mathbf{K}_{4} \Gamma(\tilde{\varepsilon}) \omega_{\mathrm{imu}} \operatorname{sgn}(\tilde{\eta})
\end{aligned}
$$

where

$$
\Gamma(\tilde{\varepsilon})=\left[\begin{array}{cccccc}
0 & 0 & \tilde{\varepsilon}_{2} & 0 & \tilde{\varepsilon}_{3} & 0 \\
\tilde{\varepsilon}_{1} & 0 & 0 & 0 & 0 & \tilde{\varepsilon}_{3} \\
0 & \tilde{\varepsilon}_{1} & 0 & \tilde{\varepsilon}_{2} & 0 & 0
\end{array}\right]^{T}
$$

and $\mathbf{K}_{i} \in \mathbb{R}^{3 \times 3}(i=1,2,3)$ and $\mathbf{K}_{4} \in \mathbb{R}^{6 \times 6}$ are gain matrices. The quaternion error $\left[\tilde{\eta}, \tilde{\varepsilon}^{T}\right]^{T}$ is obtained by combining the quaternion estimate with a reference measurement from a GPS attitude array, or other available attitude measurements. Usually, accurate roll and pitch references can be obtained from the accelerometers in inclinometer mode aided by GPS position or velocity. A high accuracy INS can also supply an accurate heading reference from gyrocompassing. GPS attitude data can be combined with the quaternion estimate either by supplying quaternions (loosely coupled), or differential phase data (tighly coupled). The error free position/velocity system is found by combining (10a), (10b), (16) and (18):

$$
\begin{aligned}
\dot{\mathbf{v}}^{n} & =\mathbf{R}_{b}^{n}\left[\left(\mathbf{I}+\Delta_{2}\right) \mathbf{f}_{\mathrm{imu}}+\mathbf{b}_{2}+\mathbf{w}_{5}\right]+\mathrm{g}^{n}-\left[2 \mathrm{~S}\left(\omega_{i e}^{n}\right)+\mathbf{S}\left(\omega_{e n}^{n}\right)\right] \mathbf{v}^{n} \\
\dot{\mathbf{r}}^{e} & =\mathbf{R}_{n}^{e} \mathbf{v}^{n} \\
\dot{\mathbf{b}}_{2} & =-\mathbf{T}_{4}^{-1} \mathbf{b}_{2}+\mathbf{w}_{6} \\
\dot{\boldsymbol{\varepsilon}} & =-\mathbf{T}_{5}^{-1} \varepsilon+\mathbf{w}_{7} \\
\dot{\boldsymbol{\beta}} & =-\mathbf{T}_{6}^{-1} \boldsymbol{\beta}+\mathbf{w}_{8}
\end{aligned}
$$




$$
\begin{aligned}
& \dot{f}=-\frac{1}{t_{7}} f+w_{9} \\
& \dot{\tau}=f+w_{10}
\end{aligned}
$$

The proposed position/velocity observer is (Vik 2000):

$$
\begin{aligned}
& \dot{\hat{\mathbf{v}}}^{n}=\hat{\mathbf{R}}_{b}^{n}\left[\left(\mathbf{I}+\hat{\Delta}_{2}\right) \mathbf{f}_{\mathrm{imu}}+\hat{\mathbf{b}}_{2}\right]+\mathbf{g}^{n}-\left[2 \mathbf{S}\left(\omega_{i e}^{n}\right)+\mathbf{S}\left(\omega_{e n}^{n}\right)\right] \hat{\mathbf{v}}^{n}+\mathbf{K}_{5} \tilde{\mathbf{v}}^{n}+\left(\mathbf{R}_{n}^{e}\right)^{T} \tilde{\mathbf{r}}^{e} \\
& \dot{\hat{\mathbf{r}}}^{e}=\mathbf{R}_{n}^{e} \hat{\mathbf{v}}^{n}+\mathbf{K}_{6} \tilde{\mathbf{r}}^{e} \\
& \dot{\hat{\mathbf{b}}}_{2}=-\mathbf{T}_{4}^{-1} \hat{\mathbf{b}}_{2}+\mathbf{K}_{7}\left(\hat{\mathbf{R}}_{b}^{n}\right)^{T} \tilde{\mathbf{v}}^{n} \\
& \dot{\hat{\varepsilon}}=-\mathbf{T}_{5}^{-1} \hat{\boldsymbol{\varepsilon}}+\mathbf{K}_{8} \operatorname{diag}\left(\mathbf{f}_{\mathrm{imu}}\right)\left(\hat{\mathbf{R}}_{b}^{n}\right)^{T} \tilde{\mathbf{v}}^{n} \\
& \dot{\hat{\boldsymbol{\beta}}}=-\mathbf{T}_{6}^{-1} \hat{\boldsymbol{\beta}}+\mathbf{K}_{9} \mathbf{Y}^{T}\left(\mathbf{f}_{\mathrm{imu}}\right)\left(\hat{\mathbf{R}}_{b}^{n}\right)^{T} \tilde{\mathbf{v}}^{n} \\
& \dot{\hat{f}}=-\frac{1}{t_{7}} \hat{f}+k_{10} \tilde{f}+\tilde{\tau} \\
& \hat{\tau}=\hat{f}+k_{11} \tilde{\tau}
\end{aligned}
$$

where

$$
\mathbf{Y}\left(\mathbf{f}_{\mathrm{imu}}\right)=\left[\begin{array}{rrrrrr}
f_{y} & f_{z} & 0 & 0 & 0 & 0 \\
0 & 0 & f_{x} & f_{z} & 0 & 0 \\
0 & 0 & 0 & 0 & f_{x} & f_{y}
\end{array}\right]^{T}
$$

$\mathbf{K}_{i} \in \mathbb{R}^{3 \times 3}(i=[5, \ldots, 8])$, and $\mathbf{K}_{9} \in \mathbb{R}^{6 \times 6}$ are positive definite gain matrices, and $k_{10}$ and $k_{11}$ are positive scalar gains. $\tilde{\mathbf{r}}^{e}, \tilde{\mathbf{v}}^{n}, \tilde{\tau}$, and $\tilde{f}$ are obtained by combining reference data from GPS with the respective estimates. In a tightly coupled system, GPS would supply pseudoranges and deltaranges, while position, velocity and clock data would be supplied in a loosely coupled system. Both of the above observers have been designed by means of a Lyapunov stability analysis.

\subsection{Stability analysis}

The nonlinear error model for the attitude observer is found from (19a) and (20):

$$
\begin{aligned}
{\left[\begin{array}{c}
\dot{\tilde{\eta}} \\
\dot{\tilde{\varepsilon}}
\end{array}\right] } & =\frac{1}{2}\left[\begin{array}{c}
-\tilde{\boldsymbol{\varepsilon}}^{T} \\
\tilde{\eta} \mathbf{I}+\mathbf{S}(\tilde{\varepsilon})
\end{array}\right]\left[\tilde{\Delta}_{1} \omega_{\mathrm{imu}}+\tilde{\mathbf{b}}_{1}-\mathbf{K}_{1} \tilde{\varepsilon} \operatorname{sgn}(\tilde{\eta})\right] \\
\dot{\tilde{\mathbf{b}}} & =-\mathbf{T}_{1}^{-1} \tilde{\mathbf{b}}_{1}-\frac{1}{2} \mathbf{K}_{2} \tilde{\varepsilon} \operatorname{sgn}(\tilde{\eta}) \\
\dot{\hat{\boldsymbol{\kappa}}} & =-\mathbf{T}_{2}^{-1} \tilde{\boldsymbol{\kappa}}-\frac{1}{2} \mathbf{K}_{3} \operatorname{diag}(\tilde{\varepsilon}) \omega_{\mathrm{imu}} \operatorname{sgn}(\tilde{\eta}) \\
\dot{\tilde{\boldsymbol{x}}} & =-\mathbf{T}_{3}^{-1} \tilde{\boldsymbol{\alpha}}-\frac{1}{2} \mathbf{K}_{4} \Gamma(\tilde{\varepsilon}) \omega_{\mathrm{imu}} \operatorname{sgn}(\tilde{\eta})
\end{aligned}
$$


By combining (21) and (22), the error dynamics for the vclocity observer takes the form:

$$
\begin{aligned}
& \dot{\hat{\mathbf{v}}}^{n}=\tilde{\mathbf{R}}_{b}^{n}\left[\tilde{\mathbf{\Delta}}_{2} \mathbf{f}_{\text {imu }}+\tilde{\mathbf{b}}_{2}\right]+\left(\mathbf{R}_{b}^{n}-\hat{\mathbf{R}}_{b}^{n}\right) \mathbf{f}-\left[2 \mathbf{S}\left(\omega_{i e}^{n}\right)+\mathbf{S}\left(\omega_{e n}^{n}\right] \tilde{\mathbf{v}}^{n}-\mathbf{K}_{5} \tilde{\mathbf{v}}^{n}-\left(\mathbf{R}_{n}^{e}\right)^{T} \tilde{\mathbf{r}}^{e}\right. \\
& \dot{\mathbf{r}}^{e}=\mathbf{R}_{n}^{e} \tilde{\mathbf{v}}^{n}-\mathbf{K}_{6} \tilde{\mathbf{r}}^{e} \\
& \dot{\dot{\mathbf{b}}}=-\mathbf{T}_{4}^{-1} \tilde{\mathbf{b}}_{2}-\mathbf{K}_{7}\left(\hat{\mathbf{R}}_{b}^{n}\right)^{T} \tilde{\mathbf{v}}^{n} \\
& \dot{\tilde{\boldsymbol{\varepsilon}}}=-\mathbf{T}_{5}^{-1} \tilde{\boldsymbol{\varepsilon}}-\mathbf{K}_{8} \operatorname{diag}\left(\mathbf{f}_{\text {imu }}\right)\left(\hat{\mathbf{R}}_{b}^{n}\right)^{T} \hat{\mathbf{v}}^{n} \\
& \dot{\tilde{\boldsymbol{\beta}}}=-\mathbf{T}_{6}^{-1} \tilde{\boldsymbol{\beta}}-\mathbf{K}_{9} \mathbf{r}\left(\mathbf{f}_{\text {imu }}\right)\left(\hat{\mathbf{R}}_{b}^{n}\right)^{T} \hat{\mathbf{v}}^{n} \\
& \dot{\tilde{\mathbf{f}}}=-t_{7}^{-1} \tilde{f}-k_{10} \tilde{f}-\tilde{\tau} \\
& \dot{\tilde{\tau}}=\tilde{f}-k_{11} \tilde{\tau}
\end{aligned}
$$

where $f=\left(I+\Delta_{2}\right) f_{i m u}+b_{2}$ is the true specific force at the navigation platform. In the following theorem we will assume that the true acceleration is bounded, i.e.

$$
\|\mathbf{f}\| \leqslant \mathbf{f}_{\max }
$$

Using the relation:

$$
\tilde{\mathbf{R}}_{b}^{n}=\mathbf{I}_{3 \times 3}+2 \tilde{\eta} \mathbf{S}(\varepsilon)+2 \mathbf{S}^{2}(\varepsilon)
$$

the expression $\left(\mathbf{R}_{b}^{n}-\hat{\mathbf{R}}_{b}^{n}\right) \mathbf{f}$ is written:

$$
\begin{aligned}
\left(\mathbf{R}_{b}^{n}-\hat{\mathbf{R}}_{b}^{n}\right) \mathbf{f} & =\hat{\mathbf{R}}_{b}^{n}(\tilde{\mathbf{R}} b-\mathbf{I}) \mathbf{f} \\
& =\hat{\mathbf{R}}_{b}^{n}\left[2 \tilde{\eta} \mathbf{S}(\tilde{\varepsilon})+2 \mathbf{S}^{2}(\tilde{\varepsilon})\right] \mathbf{f} \\
& =2 \hat{\mathbf{R}}_{b}^{n}[\tilde{\eta} \mathbf{I}+\mathbf{S}(\tilde{\varepsilon})] \mathbf{S}(\tilde{\varepsilon}) \mathbf{f} \\
& =-2 \hat{\mathbf{R}}_{b}^{n}[\tilde{\eta} \mathbf{I}+\mathbf{S}(\tilde{\varepsilon})] \mathbf{S}(\mathbf{f}) \tilde{\varepsilon} \\
& =-\mathbf{F}(\hat{\mathbf{q}}, \tilde{\mathbf{q}}, \mathbf{f}) \tilde{\varepsilon}
\end{aligned}
$$

where

$$
\mathbf{F}(\hat{\mathbf{q}}, \tilde{\mathbf{q}}, \mathbf{f})=2 \hat{\mathbf{R}}_{b}^{n}[\tilde{\eta} \mathbf{I}+\mathbf{S}(\tilde{\varepsilon})] \mathbf{S}(\mathbf{f})
$$

Theorem 1 If the condition (30) is satisfied, the total error system (23) and (24) is GES. Consider the following Lyapunov Function Candidate for (23):

$$
V_{\mathbf{1}}=\tilde{\mathbf{b}}_{1}^{T} \mathbf{K}_{w}^{-1} \tilde{\mathbf{b}}_{1}+\tilde{\boldsymbol{\kappa}}^{T} \mathbf{K}_{3}^{-1} \tilde{\boldsymbol{\kappa}}+\tilde{\alpha}^{T} \mathbf{K}_{4}^{-1} \tilde{\alpha}+\left\{\begin{array}{lll}
(\tilde{\eta}-1)^{2}+\tilde{\boldsymbol{\varepsilon}}^{T} \tilde{\varepsilon} & \text { if } & \tilde{\eta} \geqslant 0 \\
(\tilde{\eta}+1)^{2}+\tilde{\varepsilon}^{T} \tilde{\varepsilon} & \text { if } & \tilde{\eta} 0
\end{array}\right.
$$

The derivative along the trajectory of (23) is:

$$
\begin{aligned}
\dot{V}_{1} & =2 \tilde{\mathbf{b}}_{1}^{T} \mathbf{K}_{2}^{-1} \dot{\tilde{\mathbf{b}}}_{1}+2 \tilde{\boldsymbol{\kappa}}^{T} \mathbf{K}_{3}^{-1} \dot{\tilde{\boldsymbol{\kappa}}}+2 \tilde{\boldsymbol{\alpha}}^{T} \mathbf{K}_{4}^{-1} \dot{\tilde{\alpha}}+\left\{\begin{array}{rll}
-2 \dot{\hat{\eta}} & \text { if } & \tilde{\eta} \geqslant 0 \\
2 \dot{\hat{\eta}} & \text { if } & \tilde{\eta} 0
\end{array}\right. \\
& =-2 \tilde{\mathbf{b}}_{1}^{T} \mathbf{T}_{1}^{-1} \mathbf{K}_{2}^{-1} \tilde{\mathbf{b}}_{1}-2 \tilde{\boldsymbol{\kappa}}^{T} \mathbf{T}_{2}^{-1} \mathbf{K}_{3}^{-1} \tilde{\boldsymbol{\kappa}}-2 \tilde{\alpha}^{T} \mathbf{T}_{3}^{-1} \mathbf{K}_{4}^{-1} \tilde{\boldsymbol{\alpha}}-\tilde{\boldsymbol{\varepsilon}}^{T} \mathbf{K}_{1} \tilde{\varepsilon} \\
& \leqslant 0
\end{aligned}
$$


where it is used that:

$$
\begin{gathered}
\tilde{\eta} \dot{\hat{\eta}}+\tilde{\varepsilon}^{T} \hat{\varepsilon} \equiv 0 \\
\tilde{\varepsilon}^{T} \tilde{\Delta}_{1} \omega_{\mathrm{imu}}=\tilde{\kappa}^{T} \operatorname{diag}(\tilde{\varepsilon}) \omega_{\mathrm{imu}}+\tilde{\alpha}^{T} \Gamma(\tilde{\varepsilon}) \omega_{\mathrm{imu}}
\end{gathered}
$$

Let $V_{2}$ be defined as:

$$
V_{2}=\frac{1}{2}\left(\tilde{\mathbf{v}}^{n}\right)^{T} \tilde{\mathbf{v}}^{n}+\frac{1}{2}\left(\tilde{\mathbf{r}}^{e}\right)^{T} \tilde{\mathbf{r}}^{e}+\frac{1}{2} \tilde{\mathbf{b}}_{2}^{T} \mathbf{K}_{7}^{-1} \tilde{\mathbf{b}}_{2}+\frac{1}{2} \tilde{\boldsymbol{\varepsilon}}^{T} \mathbf{K}_{8}^{-1} \tilde{\boldsymbol{\varepsilon}}+\frac{1}{2} \tilde{\boldsymbol{\beta}}^{T} \mathbf{K}_{9}^{-1} \tilde{\boldsymbol{\beta}}+\frac{1}{2} \tilde{f}^{2}+\frac{1}{2} \tilde{\tau}^{2}
$$

The derivative of $V_{2}$ along (24) is:

$$
\begin{aligned}
\dot{V}_{2}= & \left(\tilde{\mathbf{v}}^{n}\right)^{T} \tilde{\mathbf{v}}^{n}+\left(\tilde{\mathbf{r}}^{e}\right)^{T} \tilde{\mathbf{r}}^{e}+\tilde{\mathbf{b}}_{2}^{T} \mathbf{K}_{7}^{-1} \dot{\tilde{\mathbf{b}}}_{2}+\tilde{\boldsymbol{\varepsilon}}^{T} \mathbf{K}_{8}^{-1} \dot{\tilde{\varepsilon}}+\tilde{\boldsymbol{\beta}}^{T} \mathbf{K}_{9}^{-1} \dot{\tilde{\boldsymbol{\beta}}}+\tilde{f} \dot{\tilde{f}}+\tilde{\tau} \tilde{\tilde{\tau}} \\
= & \left(\tilde{\mathbf{v}}^{n}\right)^{T}\left[\hat{\mathbf{R}}_{b}^{n}\left(\tilde{\Delta}_{2} \mathbf{f}_{\mathrm{imu}}+\tilde{\mathbf{b}}_{2}\right)-\left(2 \mathbf{S}\left(\omega_{i e}^{n}\right)+\mathbf{S}\left(\omega_{e n}^{n}\right)\right)^{n}-\mathbf{K}_{5} \tilde{\mathbf{v}}^{n}-\mathbf{F}(\hat{\mathbf{q}}, \tilde{\mathbf{q}}, \mathbf{f}) \tilde{\boldsymbol{\varepsilon}}\right] \\
& -\left(\tilde{\mathbf{v}}^{n}\right)^{T}\left(\mathbf{R}_{n}^{e}\right)^{T} \tilde{\mathbf{r}}^{e}+\left(\tilde{\mathbf{r}}^{e}\right)^{T}\left[\mathbf{R}_{n}^{e} \tilde{\mathbf{v}}^{n}-\mathbf{K}_{6} \tilde{\mathbf{r}}^{\prime}\right] \\
& -\tilde{\mathbf{b}}_{2}^{T}\left[\mathbf{K}_{7}^{-1} \mathbf{T}_{4}^{-1} \tilde{\mathbf{b}}_{2}+\left(\tilde{\mathbf{R}}_{b}^{n}\right)^{T} \tilde{\mathbf{v}}^{n}\right]-\tilde{\boldsymbol{\varepsilon}}^{T} \mathbf{f}_{\mathrm{imu}}\left(\hat{\mathbf{R}}_{b}^{n}\right)^{T} \tilde{\mathbf{v}}^{n} \\
& -\tilde{\boldsymbol{\beta}}^{T} \mathbf{Y}^{T}\left(\mathbf{f}_{\mathrm{imu}}\right)\left(\hat{\mathbf{R}}_{b}^{n}\right)^{T} \tilde{\mathbf{v}}^{n}-\tilde{\varepsilon}^{T} \mathbf{K}_{8}^{-1} \mathbf{T}_{5}^{-1} \tilde{\boldsymbol{\varepsilon}}-\tilde{\boldsymbol{\beta}}^{T} \mathbf{K}_{9}^{-1} \mathbf{T}_{6}^{-1} \tilde{\boldsymbol{\beta}} \\
& -\left(t_{7}^{-1}+k_{10}\right) \tilde{f}-\tilde{f} \tilde{\tau}-k_{11} \tilde{\tau}^{2}+\tilde{\tau} \tilde{f} \\
= & -\left(\tilde{\mathbf{v}}^{n}\right)^{T} \mathbf{K}_{5} \tilde{\mathbf{v}}^{n}-\left(\tilde{\mathbf{v}}^{n}\right)^{T} \mathbf{F}(\hat{\mathbf{q}}, \tilde{\mathbf{q}}, \mathbf{f}) \tilde{\boldsymbol{\varepsilon}}-\left(\tilde{\mathbf{r}}^{e}\right)^{T} \mathbf{K}_{6} \tilde{\mathbf{r}}^{e}-\tilde{\mathbf{b}}_{2}^{T} \mathbf{K}_{7}^{-1} \mathbf{T}_{4}^{-1} \tilde{\mathbf{b}}_{2} \\
& -\tilde{\boldsymbol{\varepsilon}}^{T} \mathbf{K}_{8}^{-1} \mathbf{T}_{5}^{-1} \tilde{\boldsymbol{\varepsilon}}-\tilde{\boldsymbol{\beta}}^{T} \mathbf{K}_{9}^{-1} \mathbf{T}_{6}^{-1} \tilde{\boldsymbol{\beta}}-\left(t_{7}^{-1}+k_{10}\right) \tilde{f}^{2}-k_{11} \tilde{\tau}^{2} \\
= & -\alpha(\mathbf{x}) 0 \forall \mathbf{x} \neq \mathbf{0}
\end{aligned}
$$

where $\mathbf{r}$ have been designed such that

$$
\tilde{\boldsymbol{\Lambda}}_{2} \mathbf{f}_{\mathrm{imu}}-\mathbf{Y}\left(\mathbf{f}_{\mathrm{imu}}\right) \tilde{\boldsymbol{\beta}}-\operatorname{diag}\left(\mathbf{f}_{\text {imu }}\right) \tilde{\boldsymbol{\varepsilon}}=\mathbf{0}
$$

Using $V=V_{1}+V_{2}$ as a LFC for the total system, we find that the total system is GES under the following condition:

$$
\left[\begin{array}{c}
\tilde{\mathbf{v}}^{n} \\
\tilde{\boldsymbol{\varepsilon}}
\end{array}\right]^{T}\left[\begin{array}{cc}
\mathbf{K}_{\mathbf{5}} & \frac{1}{2} \mathbf{F}(\hat{\mathbf{q}}, \tilde{\mathbf{q}}, \mathbf{f}) \\
\frac{1}{2} \mathbf{F}^{T}(\hat{\mathbf{q}}, \tilde{\mathbf{q}}, \mathbf{f}) & \mathbf{K}_{\mathbf{1}}
\end{array}\right]\left[\begin{array}{c}
\tilde{\mathbf{v}}^{n} \\
\tilde{\varepsilon}
\end{array}\right]>0
$$

By choosing $K_{1}$ and $K_{5}$ positive definite, this condition can easily be satisfied since $\|\mathbf{F}(\hat{\mathbf{q}}, \tilde{\mathbf{q}}, \mathbf{f})\|$ is bounded. Note that $V_{1}$ and thus $V$ is not strictly negative. However, with the constraint $\tilde{\eta}^{2}+\tilde{\varepsilon}^{T} \tilde{\varepsilon} \equiv 1, \dot{V}$ is strictly negative along the solution of the system. Moreover, the constraint also gives GES. This completes the proof.

\subsection{Robust stability}

The above result can be extended to include robustness to noise and other bounded disturbances and modelling errors. When noise and other bounded errors, like 
unmodeled accelerometer/gyro errors and GPS multipath, are included, the error model (23) becomes:

$$
\begin{aligned}
\dot{\tilde{q}} & =\mathbf{T}_{q}(\tilde{\mathbf{q}})\left[\tilde{\Delta}_{1} \omega_{\mathrm{imu}}+\tilde{\mathbf{b}}+\mathbf{w}_{1}-\mathbf{K}_{1}\left(\tilde{\boldsymbol{\varepsilon}}+\mathbf{H}^{\dagger} \boldsymbol{v}_{1}\right) \operatorname{sgn}(\tilde{\eta})\right] \\
\dot{\mathbf{b}}_{1} & =-\mathbf{T}_{1}^{-1} \tilde{\mathbf{b}}-\frac{1}{2} \mathbf{K}_{2}\left(\tilde{\varepsilon}+\mathbf{H}^{\dagger} \boldsymbol{v}_{1}\right) \operatorname{sgn}(\tilde{\eta})+\mathbf{w}_{2} \\
\dot{\tilde{\boldsymbol{\kappa}}} & =-\mathbf{T}_{2}^{-1} \tilde{\boldsymbol{\kappa}}-\frac{1}{2} \mathbf{K}_{3} \operatorname{diag}\left(\tilde{\varepsilon}+\mathbf{H}^{\dagger} \boldsymbol{v}_{1}\right) \omega_{\mathrm{imu}} \operatorname{sgn}(\tilde{\eta})+\mathbf{w}_{3} \\
\dot{\tilde{\alpha}} & =-\mathbf{T}_{3}^{-1} \tilde{\boldsymbol{\alpha}}-\frac{1}{2} \mathbf{K}_{4} \Gamma\left(\tilde{\varepsilon}+\mathbf{H}^{\dagger} \boldsymbol{v}_{1}\right) \omega_{\mathrm{imu}} \operatorname{sgn}(\tilde{\eta})+\mathbf{w}_{4}
\end{aligned}
$$

while the error model (24) becomes:

$$
\begin{aligned}
& \dot{\tilde{\mathbf{v}}}^{n}=\hat{\mathbf{R}}_{b}^{n}\left[\tilde{\Delta}_{2} \mathbf{f}_{\mathrm{imu}}+\tilde{\mathbf{b}}_{2}+\mathbf{w}_{5}\right]+\left(\mathbf{R}_{b}^{n}-\hat{\mathbf{R}}_{b}^{n}\right) \mathbf{f}-\left[2 \mathbf{S}\left(\omega_{i e}^{n}\right)+\mathbf{S}\left(\omega_{e n}^{n}\right)\right] \tilde{\mathbf{v}}^{n} \\
& -\mathbf{K}_{5}\left(\tilde{\mathbf{v}}^{n}+\left(\mathbf{E}^{e}\right)^{\dagger} v_{2}\right)-\left(\mathbf{R}_{n}^{e}\right)^{T}\left(\tilde{\mathbf{r}}^{e}+\left(\mathbf{E}^{e}\right)^{\dagger} \boldsymbol{v}_{3}\right) \\
& \dot{\tilde{\mathbf{r}}}^{e}=\mathbf{R}_{n}^{e} \tilde{\mathbf{v}}^{n}-\mathbf{K}_{6}\left(\tilde{\mathbf{r}}^{e}+\left(\mathbf{E}^{e}\right)^{\dagger} v_{3}\right) \\
& \dot{\tilde{\mathbf{b}}}_{2}=-\mathbf{T}_{4}^{-1} \tilde{\mathbf{b}}_{2}-\mathbf{K}_{7}\left(\hat{\mathbf{R}}_{b}^{n}\right)^{T}\left(\tilde{\mathbf{v}}^{n}+\left(\mathbf{E}^{e}\right)^{\dagger} \mathbf{v}_{2}\right)+\mathbf{w}_{6} \\
& \dot{\tilde{\varepsilon}}=-\mathbf{T}_{5}^{-1} \tilde{\varepsilon}-\mathbf{K}_{8} \operatorname{diag}\left(\mathbf{f}_{\text {imu }}\right)\left(\hat{\mathbf{R}}_{b}^{n}\right)^{T}\left(\tilde{\mathbf{v}}^{n}+\left(\mathbf{E}^{e}\right)^{\dagger} \mathbf{v}_{2}\right)+\mathbf{w}_{7} \\
& \dot{\tilde{\boldsymbol{\beta}}}=-\mathbf{T}_{6}^{-1} \tilde{\boldsymbol{\beta}}-\mathbf{K}_{9} \mathbf{Y}^{T}\left(\mathbf{f}_{\mathrm{imu}}\right)\left(\hat{\mathbf{R}}_{b}^{n}\right)^{T}\left(\tilde{\mathbf{v}}^{n}+\left(\mathbf{E}^{e}\right)^{\dagger} \boldsymbol{v}_{2}\right)+\mathbf{w}_{8} \\
& \dot{\hat{f}}=-t_{7}^{-1} \tilde{f}-k_{10}\left(\tilde{f}+\left(\mathbf{e}_{*}^{e}\right)^{\dagger} v_{2}\right)-\left(\tilde{\tau}+\left(\mathbf{e}_{*}^{e}\right)^{\dagger} v_{3}\right)+w_{9} \\
& \dot{\tilde{\tau}}=\tilde{f}-k_{11}\left(\tilde{\tau}+\left(\mathbf{e}_{*}^{e}\right)^{\dagger} v_{3}\right)+w_{10}
\end{aligned}
$$

Define the vector $\mathbf{x}=\left[\tilde{\eta}, \tilde{\varepsilon}^{T}, \tilde{\mathbf{b}}_{1}^{T}, \tilde{\boldsymbol{\kappa}}^{T}, \tilde{\alpha}^{T},\left(\tilde{\mathbf{v}}^{n}\right)^{T},\left(\tilde{\mathbf{r}}^{e}\right)^{T}, \tilde{\mathbf{b}}_{2}^{T}, \tilde{\varepsilon}^{T}, \tilde{\boldsymbol{\beta}}^{T}, \tilde{f}, \tilde{\tau}\right]^{T}$, the matrices

$$
\mathbf{Q}_{\mathbf{1}}=\left[\begin{array}{cccc}
\mathbf{K}_{1} & 0 & 0 & 0 \\
0 & 2 \mathbf{K}_{2}^{-1} \mathbf{T}_{1}^{-1} & 0 & 0 \\
0 & 0 & 2 \mathbf{K}_{3}^{-1} \mathbf{T}_{2}^{-1} & 0 \\
0 & 0 & 0 & 2 \mathbf{K}_{4}^{-1} \mathbf{T}_{3}^{-1}
\end{array}\right]
$$

$\mathbf{Q}_{2}=\left[\begin{array}{c|cccccc}\mathbf{K}_{\mathbf{5}} & 0 & 0 & 0 & 0 & 0 & 0 \\ 0 & \mathbf{I}_{\mathbf{3} \times \mathbf{3}} & 0 & 0 & 0 & 0 & 0 \\ 0 & 0 & \mathbf{K}_{7}^{-1} \mathbf{T}_{4}^{-1} & 0 & 0 & 0 & 0 \\ 0 & 0 & 0 & \mathbf{K}_{8}^{-1} \mathbf{T}_{5}^{-1} & 0 & 0 & 0 \\ 0 & 0 & 0 & 0 & \mathbf{K}_{9}^{-1} \mathbf{T}_{6}^{-1} & 0 & 0 \\ 0 & 0 & 0 & 0 & 0 & t_{7}^{-1}+k_{10} & 0 \\ 0 & 0 & 0 & 0 & 0 & 0 & k_{11}\end{array}\right]$ $\mathbf{Q}_{3}=\left[\begin{array}{ccccccc}\frac{1}{2} \mathbf{F}(\hat{\mathbf{q}}, \tilde{\mathbf{q}}, \mathbf{f}) & 0 & 0 & 0 & 0 & 0 & 0 \\ 0 & 0 & 0 & 0 & 0 & 0 & 0 \\ 0 & 0 & 0 & 0 & 0 & 0 & 0 \\ 0 & 0 & 0 & 0 & 0 & 0 & 0\end{array}\right]$ 


$$
\mathbf{Q}=\left[\begin{array}{ll}
\mathbf{Q}_{1} & \mathbf{Q}_{3} \\
\mathbf{Q}_{3}^{T} & \mathbf{Q}_{2}
\end{array}\right]
$$

and the vectors

$$
\begin{gathered}
\mathbf{n}_{1}=\left[\begin{array}{c}
\mathbf{K}_{1} \mathbf{H}^{\dagger} v_{1} \\
\mathbf{K}_{2}^{-1} \mathbf{w}_{2}+\mathbf{H}^{\dagger} v_{1} \\
\mathbf{K}_{3}^{-1} \mathbf{w}_{3}+\operatorname{diag}\left(\omega_{\text {imu }}\right) \mathbf{H}^{\dagger} v_{1} \\
\mathbf{K}_{4}^{-1} \mathbf{w}_{4}+\mathbf{r}\left(\omega_{\text {imu }}\right) \mathbf{H}^{\dagger} v_{1}
\end{array}\right] \\
\mathbf{n}_{2}=\left[\begin{array}{c}
\mathbf{K}_{5}\left(\mathbf{E}^{e}\right)^{\dagger} \boldsymbol{v}_{2}+\left(\mathbf{R}_{n}^{e}\right)^{T}\left(\mathbf{E}^{e}\right)^{\dagger} v_{3} \\
\mathbf{K}_{6}\left(\mathbf{E}^{e}\right)^{\dagger} v_{3} \\
\mathbf{K}_{7}^{-1} \mathbf{w}_{6}+\left(\hat{\mathbf{R}}_{b}^{n}\right)^{T}\left(\mathbf{E}^{e}\right)^{\dagger} v_{2} \\
\mathbf{K}_{8}^{-1} \mathbf{w}_{7}+\operatorname{diag}\left(\mathbf{f}_{\text {imu }}\right)\left(\hat{\mathbf{R}}_{b}^{n}\right)^{T}\left(\mathbf{E}^{e}\right)^{\dagger} v_{2} \\
\mathbf{K}_{9}^{-1} \mathbf{w}_{8}+\mathbf{Y}\left(\mathbf{f}_{\text {imu }}\right)\left(\hat{\mathbf{R}}_{b}^{n}\right)^{T}\left(\mathbf{E}^{e}\right)^{\dagger} v_{2} \\
k_{10}\left(\mathbf{e}_{*}^{e}\right)^{\dagger} \mathbf{v}_{2}+w_{9}+\left(\mathbf{e}_{*}^{e}\right)^{\dagger} v_{3} \\
k_{11}\left(\mathbf{e}_{*}^{e}\right)^{\dagger} v_{3}+w_{10} \\
\mathbf{n}=\left[\mathbf{n}_{1}^{T} \quad \mathbf{n}_{2}^{T}\right]^{T}
\end{array}\right]
\end{gathered}
$$

The vector $\mathbf{n}$ is bounded by

$$
\|\mathbf{n}\| \leqslant \mathbf{n}_{\max }
$$

since $w_{i}$ and $v_{i}$ are assumed bounded. The following collorary can now be proven.

Corollary 2 The solution $\mathbf{x}(t)$ of the error equations (31) and (32) is uniformly ultimately bounded with the bound $\|\mathbf{x}\| \leqslant\left\|\mathbf{Q}^{-1} \mathbf{n}_{\max }\right\|$

Following the outline of the proof of Theorem 1 above we get:

$$
\dot{V} \leqslant-\mathbf{x}^{T} \mathbf{Q} \mathbf{x}+\mathbf{x}^{T} \mathbf{n}
$$

Thus, when $\mathbf{K}_{i}, i \in[1, \ldots, 4]$ are chosen such that $\mathbf{Q}$ is positive definite, the solution $\mathbf{x}(t)$ will converge to the ball

$$
\|\mathbf{x}\| \leqslant\left\|\mathbf{Q}^{-1} \mathbf{n}_{\max }\right\|
$$

\section{Case study}

In this section, we will show the performance of the combined attitude, position and velocity observer in the case of scale-factor and bias estimation. In the simulations below, the gravity vector was fixed to give a stable vertical channel. No attempt was made to constrain the height during GPS outages. The true correlation times for the almost constant part of the biases were chosen large, that is, in the range of 10000100000 seconds. The time constants used in the observer were chosen wrong by a factor of up to ten, but the errors in the time constants had no significance during the time the simulations lasted.

Scale-factor errors can give substantial contributions to position, velocity and attitude errors during highly dynamic maneuvers. For example, if a missile is accelerat- 
Bjornar Vik and Thor I. Fossen

Table 3. Simulated IMU data

\begin{tabular}{lcl}
\hline & Accelerometers & Gyros \\
\hline Bias & $1.0 \mathrm{mg}$ & $0.002 \mathrm{rad} / \mathrm{s}$ \\
Scale-factor error & $0.03 \%$ & $0.3 \%$ \\
Noise & $0.1 \mathrm{mg}$ & $0.2 \mathrm{rad} / \sqrt{\mathrm{h}}$ \\
\hline
\end{tabular}

ing at $20 \mathrm{~m} / \mathrm{s}^{2}$ for 50 seconds with a scale-factor error of $100 \mathrm{ppm}$, the resulting velocity error will be $0.1 \mathrm{~m} / \mathrm{s}$, while the position error will be $2.5 \mathrm{~m}$. This is not very important in cases where continuous GPS measurements are available. But in the case of GPS outage during maneuvers, the errors will in general build up for each maneuver. The exception to this is applications with symmetric or periodic maneuvers, since errors will cancel out. In this case, only scale-factor unsymmetry will be a factor.

Scale-factor errors are only observable during acceleration or attitude changes. The best way of estimating them, is to perform certain maneuvers on purpose. In this way, the filter can be tuned to get the best estimate for the time a given maneuver lasts. It is also an advantage to estimate biases to a certain accuracy beforehand, something which can be accomplished in most cases, since no maneuvering is needed to observe the biases. If biases are not estimated beforehand, more time and maneuvers are necessary to estimate biases and scale-factors simultaneously.

To show the effect of scale-factor errors, we simulated a vehicle under course changing maneuvers. In the simulations, data from a low cost IMU shown in Table 3, was used. Accelerometer scale-factor errors were not estimated, since the effect of them were minor. The trajectory was repeated after $200 \mathrm{~s}$, and lock is lost on all satellites for the last 200 seconds. The maneuvers started at 40 seconds, which gave time for the gyro biases to converge before the scale-factor errors became observable. Figure 3 shows the PVA errors that built up during the outage when bias estimation was included, but scale-factor error estimation was omitted. The error that builds up in attitude the first 20 seconds is due to gyro bias, while the error in yaw after about 70 seconds is due to the yaw rate scale-factor error.

For the case where scale-factor errors were estimated, the PVA response is shown in Figure 4. A clear difference in heading error can be seen, in addition to a smaller error in position due to the more accurate heading. The gyro bias and scale-factor error estimates are shown in Figure 5. Due to very little roll and pitch motions, only the yaw rate scale-factor error estimate has converged close to the true value.

\section{Conclusions}

In this paper, some of the features of integrated inertial and satellite navigation systems are described. A combined attitude and position/velocity observer for the purpose of integration has been presented. The observer is suitable for direct integration architectures, as opposed to the extended Kalman-filter. Compared to the latter, the observer has major computational advantages in a direct architecture. The direct approach is, in our opinion, more intuitive and less complex than the indirect approach employed by most other designs, and the observer is also a little less computationally demanding than these. The origin of the observer error-system has been proven globally exponentially stable, while the popular extended Kalman-filter only has been proven locally exponentially stable. Finally, robust stability in the presence of noise and unmodelled effects have been proven. 

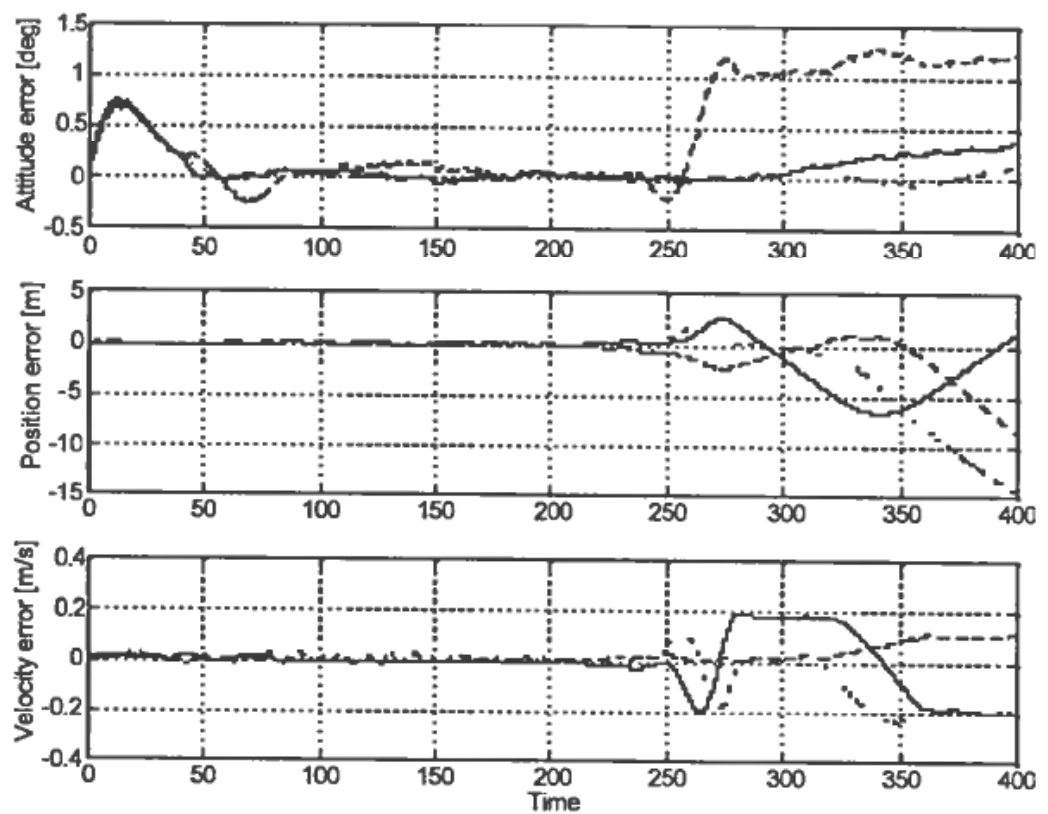

Figure 3. Attitude, ECEF position, and NED velocity errors when scale-factor errors are not estimated. The solid lines are the first axes (roll, ECEF x-axis, and north respectively), the dotted lines are the second axes, and the dashed lines are the third axes. No satellite measurements were available from $200-400 \mathrm{~s}$.
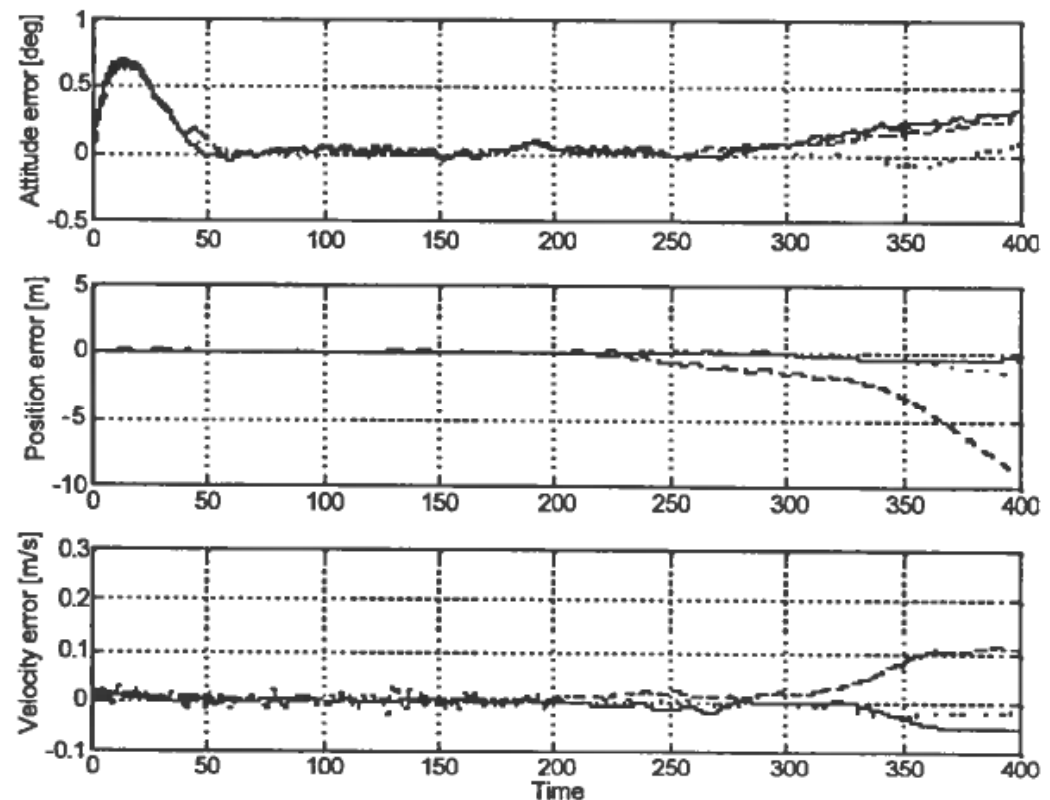

Figure 4. Attitude, ECEF position, and NED velocity errors when scale-factor errors are estimated. The solid lines are the first axes (roll, ECEF x-axis, and north respectively), the dotted lines are the second axes, and the dashed lines are the third axes. No satellite measurements were available from $200-400 \mathrm{~s}$ 

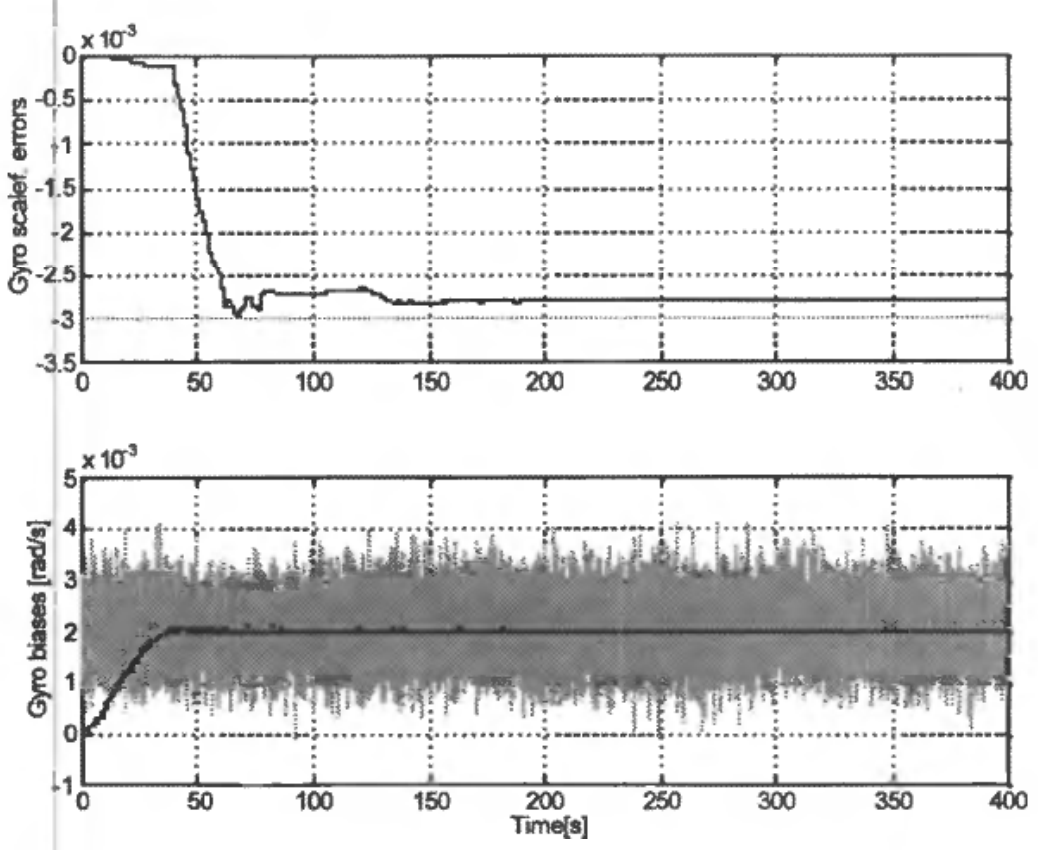

Figure 5. Upper plot: Gyro scale-factor errors in black, and true scale-factor errors in grey. Yaw rate error is solid, while roll and pitch rate errors are dashed and dotted respectively. Lower plot: Gyro bias estimates are in black, while the true values + gyronoise is in grey. Estimation is turned off at $200 \mathrm{~s}$ when GPS measurements are lost.

\section{REFERENCES}

Britting, K. R. (1971). Inertial Navigation Systems Analysis, Wiley Interscience.

Greenspan, R. L., Donna, J. I. and Soltz, J. A. (1988). The GPS Users Integration Guide. In: Proceedings of National Technical Meeting ION, pp. 104-112.

Hofmann-Wellenhof, B., Lichtenegger, H. and Collins, J. (1994). Global Positioning System: Theory and Practice, Springer Verlag, Wien, New York.

Lakshmikantham, V., Matrosov, V. M. and Sivasundaram, S. (1991). Vector Lyapunov Functions and Stability Analysis of Nonlinear Systems, Kluwer Academic Publishers.

Maybeck, P. S. (1979). Stochastic Models, Estimation and Control Vol. 1, Academic Press, New York.

PhILliPS, R. E. and SCHMidT, G. T. (1996). GPS/INS Integration, AGARD Lecture Series 207: System Implications and Innovative Applications of Satellite Navigation, pp. 9.1-9.18.

VIK, B. (2000). Nonlinear Design and Analysis of Integrated GPS and Inertial Navigation Systems. PhD thesis, Norwegian University of Science and Technology.

VIK, B., ShIRIAEV, A. and Fossen, T. I. (1999). Nonlinear Observer Design for Integration of DGPS and INS. In: New Directions in Nonlinear Observer Design (H. NiJMEIJER and T. I. FosSEN, Eds), p. 135, Springer-Verlag Ltd, London. 